\title{
TWOPRIME: A Fast Stream Ciphering Algorithm
}

\author{
Cunsheng Ding1, Valtteri Niemi ${ }^{2}$, Ari Renvall ${ }^{3}$, Arto Salomaa ${ }^{3}$ \\ 1 Turku Centre for Computer Science, Datacity 4th floor, 20520 Turku, Finland \\ 2 Department of Mathematics \& Statiscias, University of Vaasa, FIN-65101 Vaasa, \\ Finland \\ 3 Department of Mathematics, University of Turku, FIN-20014 Turku, Finland
}

\begin{abstract}
In this paper, we describe an additive stream ciphering algorithm, called "TWOPRIME". It is designed for 32-bit computers, and the key has 128 bits. It is fast in software and analytical in the sense that some security aspects of the algorithm can be controlled. A faster version of TWOPRIME is also presented. We also describe a variant of TWOPRIME, called ONEPRIME, which is for 64-bit machines.
\end{abstract}

\section{Introduction}

There are a number of ciphering algorithms, but most of them are limited to a few models such as the Feistel network. The purpose of this paper is to describe a ciphering algorithm that is different to known ones in the public domain with the following properties:

- its structure should be special;

- the key size should be large enough;

- it is for 32-bit computers;

- it should work on blocks of bytes;

- it should be fast in software;

- it should be analytical;

- it should be easily modified for 64 -bit computers;

- it is expected to be secure.

TWOPRIME is an additive synchronous stream cipher that is nonproprietary. It has a 128-bit key, and the encryption and decryption algorithms are the same. A C code runs at $1 \mathrm{Mbytes} / \mathrm{sec}$ on a Pentium $(75 \mathrm{MHz})$. It should be faster than a number of fast ciphers in the public domain (but looks slower than SEAL [8]). The main feature of our algorithm is that the period, linear complexity, and distributions of some patterns of the keystream sequences are controllable. Compared with other stream ciphers, TWOPRIME seems analytical.

In this paper, we also present a faster version of TWOPRIME. A C code of this faster version runs at $1.3 \mathrm{Mbytes} / \mathrm{sec}$ on the same Pentium. As a variant of TWOPRIME, we describe the ONEPRIME ciphering algorithm for 64-bit computers, which should be much faster than TWOPRIME. 


\section{TWOPRIME: a description}

This additive stream cipher works on blocks of 8 bytes. The keystream generator produces an 8-byte keystream block at each time unit, and this keystream block is then bytewise exored with the 8-byte block of input.

The key of the algorithm has 16 bytes, denoted by $k_{0} k_{1} \cdots k_{15}$, which are divided into four parts. Let

$$
\begin{aligned}
& K_{0}=k_{8}+k_{9} 2^{8}+k_{10} 2^{16}+k_{11} 2^{24}, \\
& K_{1}=k_{12}+k_{13} 2^{8}+k_{14} 2^{16}+k_{15} 2^{24}, \\
& K_{2}=\left(k_{0}, k_{1}, k_{2}, k_{3}\right), \\
& K_{3}=\left(k_{4}, k_{5}, k_{6}, k_{7}\right) .
\end{aligned}
$$

The algorithm has ten layers. The first layer consists of two $(p, a)$ cyclic counters. A $(p, a)$ cyclic counter has an internal register that can store any integer between 0 and $p-1$, thus the register has $\left\lceil\log _{2} p\right\rceil$ bits of memory. The initial value of the register is an integer $k$, where $0 \leq k \leq p-1$. The value of the register at time unit $i$ is defined to be

$$
r_{i}=(a i+k \bmod p),
$$

where $z \bmod m$ denotes the least nonnegative integer that is congruent to $z$ modulo $m$, where $\operatorname{gcd}(a, p)=1$. When $a=1$, it counts the numbers $k,(k+$ 1) $\bmod p, \cdots,(k+p-1 \bmod p)$ cyclically. When $a \neq 1 \bmod p$, we call it a cyclic counter with step $a$ and period $p$, in other words, a $(p, a)$ cyclic counter.

In the two $\left(p_{i}, a_{i}\right)$ cyclic counters, $p_{0}$ and $p_{1}$ are two distinct primes having 32 bits, and $a_{0}$ and $a_{1}$ are two constants between 0 and $p_{i}-1$ respectively. The largest two 32 -bit primes are $p_{1}=4294967291$ and $p_{0}=4294967279$. Note that

$$
p_{1}-1=2 \times 5 \times 19 \times 22605091, p_{0}-1=2 \times 7 \times 17 \times 18046081 .
$$

It has been computed that

$$
\begin{array}{ll}
\left(2^{18046081} \bmod p_{0}\right) & =1145473156, \\
\left(2^{7 \times 18046081} \bmod p_{0}\right) & =2366705928, \\
\left(2^{17 \times 18046081} \bmod p_{0}\right) & =1145324610, \\
\left(2^{\left(p_{1}-1\right) / 2} \bmod p_{0}\right) & =1, \\
\left(2^{7 \times 17} \bmod p_{0}\right) & =2558525593 .
\end{array}
$$

Hence the order of 2 modulo $p_{0}$ is

$$
\operatorname{ord}_{p_{0}}(2)=\left(p_{0}-1\right) / 2=2147483639=2^{31}-9 .
$$

We have also computed that

$$
\begin{array}{ll}
\left(2^{22605091} \bmod p_{1}\right) & =3079820090 \\
\left(2^{5 \times 22605091} \bmod p_{1}\right) & =3786472082 \\
\left(2^{19 \times 22605091} \bmod p_{1}\right) & =1304151046 \\
\left(2^{\left(p_{1}-1\right) / 2} \bmod p_{1}\right) & =p_{1}-1 \\
\left(2^{5 \times 19} \bmod p_{1}\right) & =2147483708
\end{array}
$$




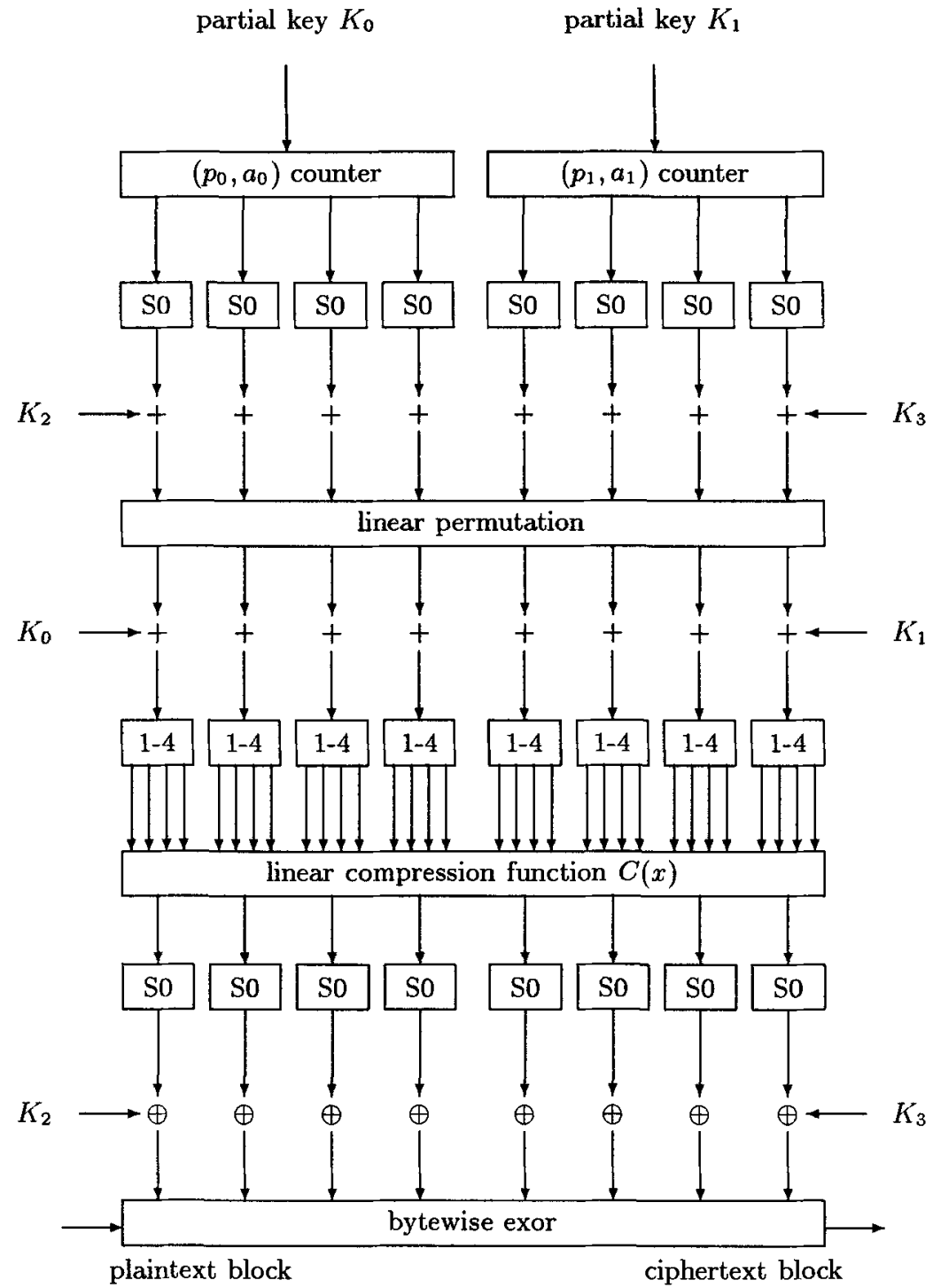

Fig. 1. Structure of the ciphering algorithm.

Hence

$$
\operatorname{ord}_{p_{1}}(2)=p_{1}-1=2^{32}-6
$$

Thus, 2 is a primitive root modulo $p_{1}$. We have seen that the two primes are almost equal to $2^{32}-1$ such that the orders of 2 modulo them are close to $2^{31}$.

The two constants $a_{i}$ are chosen such that 
1. $a_{i}>\left(p_{i}-1\right) / 2$ for $i=0$ and 1 , in order that in every two consecutive two updatings of the two registers of the two cyclic counters in the keystream generator there is one modulo- $p_{i}$ reduction;

2. they should be different and the difference should be large enough;

3. each $a_{i}$ should not be too close to $p_{i}$;

4. they are primes (this leads to $\operatorname{gcd}\left(a_{1}, a_{2}\right)=1$ ).

Basing on the above considerations we suggest the following two constants:

$$
a_{0}=2345986071, \quad a_{1}=3124567807 \text {. }
$$

Of course, there are many such choices for the constants $a_{i}$.

The second layer of the algorithm consists of eight S-boxes $S 0$ that is a permutation of $Z_{256}$ with good nonlinearity with respect to the addition of the residue class ring $Z_{256}$. The first, second, third, and fourth bytes of the contents of the two registers of the two cyclic counters are used as the inputs of the eight $\mathrm{S}$-boxes $S 0$. This is the first nonlinear layer.

The nonlinear permutation $S 0$ is defined by

$$
S 0(x)=\left[\left(x^{255} \bmod 257\right) \bmod 256\right], \quad x \in Z_{256} .
$$

The permutation $x^{255}=x^{-1}$ has good nonlinearity with respect to the addition of $Z_{257}$ (It is well-known that $X^{-1}$ is a permutation of finite fields with good nonlinearity, and it has been used in the cipher Shark [6]). Computation proves that the above permutation $S 0$ has also good nonlinearity with respect to the addition of $Z_{256}$. The approach to finding a good nonlinear permutation of $Z_{256}$ follows that used by Massey [5].

The third layer is the bytewise addition of the outputs of the second round and the partial keys $K_{2}$ and $K_{3}$. The outputs of the first four S-boxes $S 0$ are added to the four bytes of $K_{2}$, and those of the second four S-Boxes $S_{0}$ are added to the four bytes of $K_{3}$, where all additions are that of $Z_{256}$, i.e., integer addition modulo 256.

The fourth layer is a linear one that is for diffusion of the key. Let $X_{0}, \cdots, X_{7}$ be the eight input bytes of this layer, its eight output bytes are defined by

$$
Y_{j}=\sum_{i=0}^{7} X_{i}-X_{j}, j=0,1, \cdots, 7,
$$

where "+" and " - " denote the addition and subtraction of $Z_{256}$. It is clear that the change of one byte leads to a change of seven of the eight output bytes of this layer. This is a relatively expensive layer, compared with others.

The fifth layer is again a key-addition layer, but this time the partial keys $K_{0}$ and $K_{1}$ are added. This makes it difficult to find some key-equivalence classes, by which we mean that they determine the same encryption transformation.

The sixth layer is nonlinear and also for data expansion. It has eight-byte inputs, but 32-byte outputs. Each box containing a symbol 1-4 denotes an array 
of four S-boxes in the order S1, S2, S3, S4. The four S-boxes are defined by

$$
\begin{aligned}
& S 1(x)=\left[x^{3} \bmod 257\right] \bmod 256, \\
& S 2(x)=\left[x^{171} \bmod 257\right] \bmod 256, \\
& S 3(x)=\left[45^{x} \bmod 257\right] \bmod 256, \\
& S 4(x)= \begin{cases}{\left[\log _{45} x \bmod 257\right] \bmod 256,} & \text { if } x \neq 0 ; \\
128, & \text { if } x=0 .\end{cases}
\end{aligned}
$$

S3 and S4 are the two S-boxes used in SAFER [5]. As far as nonlinearity is concerned, S3 and S4 are the best nonlinear permutations of $Z_{256}$ with respect to the addition of $Z_{256} . S 1$ and $S 2$ have also good nonlinearity, but not as good as $S 3$ and $S 4$. In fact, $S 1$ and $S 2$ have the same nonlinearity as $S 0$. However, it should be mentioned that the nonlinearity with respect to the bytewise exor of $S 1$ and $S 2$ is much better than that of $S 3$ and $S 4$. One should be careful to use $S 3$ and $S 4$ with respect to bytewise exor operation. This is seen below.

The nonlinearity or differentiality of a permutation $P(x)$ of $Z_{256}$ with respect to the addition of $Z_{256}$ is measured by the probability

$$
\operatorname{Pr}(P(x+a)-P(x)=b) .
$$

When $a=0$, this probability is 1 or 0 and it is not interesting in any attack. So we are only interested in the case $a \neq 0$. Note that if $P(x)$ is a permutation, the equation $P(x+a)-P(x)=0$ has no solution. So for any fixed $a \neq 0$ we have

$$
\max _{b \neq 0} \operatorname{Pr}(P(x+a)-P(x)=b) \geq 2 / 256=1 / 128 \text {. }
$$

Hence

$$
\begin{aligned}
\frac{1}{128} & \leq \max _{b \neq 0} \operatorname{Pr}(P(x+a)-P(x)=b) \leq \\
& \leq \max _{a \neq 0} \max _{b \neq 0} \operatorname{Pr}(P(x+a)-P(x)=b) .
\end{aligned}
$$

For some cryptographic applications the smaller the $\max _{a \neq 0} \max _{b \neq 0} \operatorname{Pr}(P(x+$ a) $-P(x)=b)$ the better the security with respect to some attacks. If the two equalities of $(2)$ hold, we say that the permutation $P(x)$ has the best nonlinearity or differentiality with respect to the addition of $Z_{256}$.

The two permutations $S 3(x)$ and $S 4(x)$ have the best nonlinearity or differentiality with respect to the addition of $Z_{256}$. This has been proved by a $\mathrm{C}$ program.

It should be noted that the two permutations $S 3(x)$ and $S 4(x)$ have bad nonlinearity or differentiality with respect to the bytewise EXOR operation. This is justified by the following formulae:

$$
\operatorname{Pr}(S 3(x \oplus 128) \oplus S 3(x)=b)=\left\{\begin{array}{l}
\frac{1}{2}, \quad b=253 ; \\
\frac{1}{4}, \quad b=249 ; \\
\frac{1}{8}, \quad b=241 ; \\
\frac{1}{16}, b=225 \\
\frac{1}{32}, b=213 \\
\frac{1}{64}, b=129 ; \\
\frac{1}{64}, b=1 ; \\
0 ; \text { otherwise. }
\end{array}\right.
$$


Also we have

$$
\operatorname{Pr}(S 3(x \oplus 64) \oplus S 3(x)=b) \in\left\{\frac{22}{256}, \frac{18}{256}, \frac{10}{256}, \frac{8}{256}, \frac{6}{256}, \frac{4}{256}, \frac{2}{256}, 0\right\},
$$

where " $\oplus$ " is the bytewise exor.

These formulae show clearly that the two permutations $S 3(x)$ and $S 4(x)$ have bad nonlinearity or differentiality with respect to the bytewise exor. Thus, one has to be careful in using the two permutations. However, in our algorithm we have used the two permutations only with respect to the addition of $Z_{256}$.

The seventh layer is a linear compression one, which has 32-byte inputs and eight-byte outputs. We denote the inputs of this layer from the left to the right by $X_{0}, X_{1}, \cdots, X_{31}$, and the outputs from the left to the right by $Y_{0}, Y_{1}, \cdots, Y_{7}$. Then the linear compression function $C(x)$ is defined by

$$
\left\{\begin{array}{l}
Y_{0}=X_{0}+X_{5}+X_{10}+X_{15}+X_{16}+X_{22}+X_{24}+X_{30} \\
Y_{1}=X_{1}+X_{6}+X_{11}+X_{12}+X_{17}+X_{23}+X_{25}+X_{31}, \\
Y_{2}=X_{2}+X_{7}+X_{8}+X_{13}+X_{18}+X_{20}+X_{26}+X_{28}, \\
Y_{3}=X_{3}+X_{4}+X_{9}+X_{14}+X_{19}+X_{21}+X_{27}+X_{29}, \\
Y_{4}=X_{16}+X_{21}+X_{26}+X_{31}+X_{0}+X_{6}+X_{8}+X_{14}, \\
Y_{5}=X_{17}+X_{22}+X_{27}+X_{28}+X_{5}+X_{11}+X_{13}+X_{3} \\
Y_{6}=X_{18}+X_{23}+X_{24}+X_{29}+X_{10}+X_{12}+X_{2}+X_{4} \\
Y_{7}=X_{19}+X_{20}+X_{25}+X_{30}+X_{15}+X_{1}+X_{7}+X_{9},
\end{array}\right.
$$

Thus, every output byte depends on 8 input bytes, and every input byte affects two output bytes. This linear function is mainly for compression, but it also plays an important role in diffusion. The data expansion and compression are designed to prevent one from inverting the whole system backwards. This also makes each output byte dependable on as many S-boxes and inputs of the expansion layer as possible.

The eighth layer is a nonlinear one, where eight $\mathrm{S}$-boxes $\mathrm{S} 0$ are applied. The ninth layer is again a key-addition one, but here the addition is bytewise exor. This is also designed to prevent one from going backwards to the front of the keystream generator. The last layer is the bytewise exor of the keystream block and the plaintext block.

\section{Theoretical results about the algorithm}

Let $R$ be a commutative ring with multiplicative identity 1 , and

$$
s^{N}=s_{0} s_{1} \cdots s_{N-1}
$$

be a sequence of length $N$ over $R$, where $s_{i} \in R$. If $s^{N}$ satisfies a linear recurrence relation

$$
s_{i}=a_{1} s_{i-1}+a_{2} s_{i-2}+\cdots+a_{l} s_{i-l}, \quad i \geq l, a_{i} \in R
$$


then there exists such a shortest linear recurrence relation, and the shortest $l$ is called the linear complexity or linear span of the sequence and is denoted by $\mathrm{L}\left(s^{N}\right)$.

If the linear complexity of a sequence over a field is $l$, then $2 l$ successive characters of the sequence can be used to determine a linear recurrence relation of length $l$ satisfied by the sequence with the Berlekamp-Massey algorithm [4], which has complexity $O\left(l^{2}\right)$. Thus, $2 l$ successive characters of the sequence are sufficient to determine the whole sequence. Thus, sequences over fields for additive stream ciphers should have large linear complexity.

For sequences over $Z_{m}$, which is the ring $\{0,1, \ldots, m-1\}$ with integer addition modulo $m$ and multiplication modulo $m$, the Berlekamp-Massey algorithm does not work, but the Reeds-Sloane algorithm works. The latter is an analog of the Berlekamp-Massey algorithm, and it is also efficient [7]. Thus, it is necessary to control the linear complexity of sequences over $Z_{m}$ for additive stream ciphering.

To prove some theoretical results about the algorithm, we need the following lemma [3]:

Lemma 1. Let $N=p_{1}^{e_{1}} \cdots p_{t}^{e_{t}}$, where $p_{1}, \cdots, p_{t}$ are $t$ pairwise distinct primes, $q$ an integer such that $\operatorname{gcd}(q, N)=1$. Then for each nonconstant sequence $s^{\infty}$ of period $N$ over $G F(q)$,

$$
\mathrm{L}\left(s^{\infty}\right) \geq \min \left\{\operatorname{ord}_{p_{1}}(q), \cdots, \operatorname{ord}_{p_{t}}(q)\right\},
$$

Where $\mathrm{L}$ denotes the linear complexity.

Proposition 2. Concerning the keystream generator we have the following conclusions:

1. Each output sequence of bytes has least period $p_{0}, p_{1}$ or $p_{0} p_{1}$.

2. Each output sequence of bytes over the ring $Z_{256}$ has linear complexity at least $\min \left\{\operatorname{ord}_{p_{0}}(2), \operatorname{ord}_{p_{1}}(2)\right\}=2^{31}-9$.

3. The elements of $Z_{256}$ are almost equally likely distributed in a cycle of each output sequence of bytes.

4. All the above conclusions hold for each output bit sequence.

Proof: Note that the output sequence of the register of the $\left(p_{0}, a_{0}\right)$ (resp. $\left.\left(p_{1}, a_{1}\right)\right)$ has least period $p_{0}$ (resp. $p_{1}$ ). Let $X_{1}, X_{2}, X_{3}, X_{4}$ be the four output bytes of the $\left(p_{0}, a_{0}\right)$ cyclic counter at each time unit, and $X_{5}, X_{6}, X_{7}, X_{8}$ be those of the four output bytes of the $\left(p_{1}, a_{1}\right)$ cyclic counter. It follows that the semi-infinite sequences $X_{i}^{\infty}$ have least period $p_{0}$ for $i=1,2,3,4$, and $p_{1}$ for $i=5,6,7,8$.

Let $Y_{1}=X_{2}+X_{3}+X_{4}+X_{5}+X_{6}+X_{7}+X_{8}$ mod 256. Then the semi-infinite sequence $Y_{1}^{\infty}$ has period $p_{0} p_{1}$. It follows that its least period must be one of $1, p_{0}, p_{1}, p_{0} p_{1}$. Obviously, $Y_{1}^{\infty}$ is not a constant sequence. Thus, its least period cannot be 1. Suppose that the least period of $Y_{1}^{\infty}$ is $p_{0}$. Then the semi-infinite sequence $\left(X_{5}+X_{6}+X_{7}+X_{8}\right)^{\infty}$ must have a period $p_{0}$, but it has a period $p_{1}$. This is impossible since $p_{0}$ and $p_{1}$ are distinct primes. Hence, the semi-infinite sequence $Y_{1}^{\infty}$ must have least period $p_{0} p_{1}$. The same conclusion holds for $Y_{i}^{\infty}$, 
where $2 \leq i \leq 8$. Since each output byte (bit) sequence cannot be a constant sequence, the least period of each output sequence should be one of $\left\{p_{0}, p_{1}, p_{0} p_{1}\right\}$.

We have already proven that each output bit sequence has a period (not necessary the least one) $p_{0} p_{1}$. By Lemma 1 , the linear complexity of each output bit sequence is at least $\min \left\{\operatorname{ord}_{p_{0}}(2), \operatorname{ord}_{p_{1}}(2)\right\}$.

Let $z_{1}^{\infty}=Z_{1}^{\infty} \bmod 2$, where $Z_{1}^{\infty}$ is the output byte sequence of the first output byte position of the keystream generator, it is easily seen that the linear complexity of the semi-infinite sequence $z_{1}^{\infty}$ over $Z_{256}$ is no less than that of $Z_{1}^{\infty}$. Thus, we have proved the second claim.

If $p_{0}=p_{1}=2^{32}$, then each $X_{i}$ takes on elements of $Z_{256}$ equally likely, so does each $Y_{i}$. However, since $p_{0}=2^{32}-17$ and $p_{1}=2^{32}-5$, each output byte $Y_{i}$ takes on elements of $Z_{256}$ almost equally likely. Since each layer is either a permutation layer or a linear layer, each keystream byte sequence has an almost equally likely distribution of the elements of $Z_{256}$, so each bit sequence of the keystream block sequence has an almost equally likely distribution of ones and zeroes.

Remark: It should be extremely unlikely that the least period of a byte (bit) sequence is $p_{0}$ or $p_{1}$.

\section{Security arguments}

A cipher must be secure against ciphertext-only attacks if it is secure against known-plaintext attacks. So in the sequel we shall argue some security aspects of the algorithm only with respect to some known plaintext attacks. When doing so, we assume the cryptanalyst has sufficiently many keystream blocks. Similar to other practical ciphers, it is hard to prove the security of a ciphering algorithm since we cannot sort out all possible attacks on a cipher.

\section{With respect to brute-force attack}

An attack that applies to every cipher is the brute-force attack by trying all possible keys. Since the number of possible keys of our ciphering algorithm is $2^{128}$, this attack should not work. On the other hand, it might be possible that a number of keys determine the same encryption transformation, but we do not see a way to prove their existence, let alone to determine them if they exist.

\section{With respect to linear complexity attacks}

Since this is an additive synchronous stream cipher, it is necessary to control the least period (cycle length) of the keystream sequences and its component bit sequences. As proved before, the least period of the output sequence and its component bit sequences all have least period $>\min \left\{p_{0}, p_{1}\right\}$, and the linear complexities of the output sequence and its component bit sequences are at least

$$
\min \left\{\operatorname{ord}_{p_{0}}(2), \operatorname{ord}_{p_{1}}(2)\right\}=2^{31}-9 .
$$


Thus, any attack based on the Berlekamp-Massey algorithm [4] or Reeds-Sloane algorithm [7] should not work. We can also prove that the linear complexities of the output sequence and its component bit sequences have ideal stability, thus, it is hard to construct an LFSR to approximate the output sequence of the generator $[2]$.

\section{With respect to inverting attacks}

One basic question is whether this key-stream generator is invertible. Except the data-expansion layer and the data-compression layer, all other layers are permutation layers, when the key is fixed. But without the key it could be impossible to invert the keystream generator.

Let $W=\left(W_{0}, W_{1}, \cdots, W_{7}\right)$ be a keystream block and let $Y=\left(Y_{0}, Y_{1}, \cdots, Y_{7}\right)$ be the corresponding output of the linear compression layer. Then we have

$$
W_{i}=k_{i} \oplus S 0\left[Y_{i}\right], \quad i=0,1, \cdots, 7,
$$

where the addition is the bytewise exor. Assuming that the key is randomly chosen, the information about $W$ provided by the keystream block is zero. Thus, it is impossible to use one keystream block to go backwards.

Note that the last layer of the keystream generator is linear with respect to bytewise exor, one may consider the difference of two keystream blocks, in order to get rid of the partial keys $K_{2} K_{3}$ added. We now analyze what we can get from this idea.

Let $W=\left(W_{0}, \cdots, W_{7}\right)$ and $W^{\prime}=\left(W_{0}^{\prime}, \cdots, W_{7}^{\prime}\right)$ be two keystream blocks at time $t$ and time $t^{\prime}$, and let $\left(Z_{0}, \cdots, Z_{7}\right)$ and $\left(Z_{0}^{\prime}, \cdots, Z_{7}^{\prime}\right)$ be the corresponding inputs of the data-expansion layer. Then

$$
\begin{array}{r}
W_{0} \oplus W_{0}^{\prime}=S 0\left[S 1\left(Z_{0}\right)+S 2\left(Z_{1}\right)+S 3\left(Z_{2}\right)+S 4\left(Z_{3}\right)+\right. \\
\left.S 1\left(Z_{4}\right)+S 3\left(Z_{5}\right)+S 1\left(Z_{6}\right)+S 3\left(Z_{7}\right)\right] \oplus \\
S 0\left[S 1\left(Z_{0}^{\prime}\right)+S 2\left(Z_{1}^{\prime}\right)+S 3\left(Z_{2}^{\prime}\right)+S 4\left(Z_{3}^{\prime}\right)+\right. \\
\left.S 1\left(Z_{4}^{\prime}\right)+S 3\left(Z_{5}^{\prime}\right)+S 1\left(Z_{6}^{\prime}\right)+S 3\left(Z_{7}^{\prime}\right)\right], \\
W_{1} \oplus W_{1}^{\prime}=S 0\left[S 2\left(Z_{0}\right)+S 3\left(Z_{1}\right)+S 4\left(Z_{2}\right)+S 1\left(Z_{3}\right)+\right. \\
\left.S 2\left(Z_{4}\right)+S 4\left(Z_{5}\right)+S 2\left(Z_{6}\right)+S 4\left(Z_{7}\right)\right] \oplus \\
S 0\left[S 2\left(Z_{0}^{\prime}\right)+S 3\left(Z_{1}^{\prime}\right)+S 4\left(Z_{2}^{\prime}\right)+S 1\left(Z_{3}^{\prime}\right)+\right. \\
\left.S 2\left(Z_{4}^{\prime}\right)+S 4\left(Z_{5}^{\prime}\right)+S 2\left(Z_{6}^{\prime}\right)+S 4\left(Z_{7}^{\prime}\right)\right], \\
W_{2} \oplus W_{2}^{\prime}=S 0\left[S 3\left(Z_{0}\right)+S 4\left(Z_{1}\right)+S 1\left(Z_{2}\right)+S 2\left(Z_{3}\right)+\right. \\
\left.S 3\left(Z_{4}\right)+S 1\left(Z_{5}\right)+S 3\left(Z_{6}\right)+S 1\left(Z_{7}\right)\right] \oplus \\
S 0\left[S 3\left(Z_{0}^{\prime}\right)+S 4\left(Z_{1}^{\prime}\right)+S 1\left(Z_{2}^{\prime}\right)+S 2\left(Z_{3}^{\prime}\right)+\right. \\
\left.S 3\left(Z_{4}^{\prime}\right)+S 1\left(Z_{5}^{\prime}\right)+S 3\left(Z_{6}^{\prime}\right)+S 1\left(Z_{7}^{\prime}\right)\right], \\
W_{3} \oplus W_{3}^{\prime}=S 0\left[S 4\left(Z_{0}\right)+S 1\left(Z_{1}\right)+S 2\left(Z_{2}\right)+S 3\left(Z_{3}\right)+\right. \\
\left.S 4\left(Z_{4}\right)+S 2\left(Z_{5}\right)+S 4\left(Z_{6}\right)+S 2\left(Z_{7}\right)\right] \oplus \\
S 0\left[S 4\left(Z_{0}^{\prime}\right)+S 1\left(Z_{1}^{\prime}\right)+S 2\left(Z_{2}^{\prime}\right)+S 3\left(Z_{3}^{\prime}\right)+\right. \\
\left.S 4\left(Z_{4}^{\prime}\right)+S 2\left(Z_{5}^{\prime}\right)+S 4\left(Z_{6}^{\prime}\right)+S 2\left(Z_{7}^{\prime}\right)\right],
\end{array}
$$




$$
\begin{array}{r}
W_{4} \oplus W_{4}^{\prime}=S 0\left[S 1\left(Z_{4}\right)+S 2\left(Z_{5}\right)+S 3\left(Z_{6}\right)+S 4\left(Z_{7}\right)+\right. \\
\left.S 1\left(Z_{0}\right)+S 3\left(Z_{1}\right)+S 1\left(Z_{2}\right)+S 3\left(Z_{3}\right)\right] \oplus \\
S 0\left[S 1\left(Z_{4}^{\prime}\right)+S 2\left(Z_{5}^{\prime}\right)+S 3\left(Z_{6}^{\prime}\right)+S 4\left(Z_{7}^{\prime}\right)+\right. \\
\left.\left.S 1\left(Z_{0}^{\prime}\right)+S 3\left(Z_{1}^{\prime}\right)+S 1\left(Z_{2}^{\prime}\right)+S 3\left(Z_{3}^{\prime}\right)\right]\right], \\
W_{5} \oplus W_{5}^{\prime}=S 0\left[S 2\left(Z_{4}\right)+S 3\left(Z_{5}\right)+S 4\left(Z_{5}\right)+S 1\left(Z_{5}\right)+\right. \\
\left.S 2\left(Z_{1}\right)+S 4\left(Z_{2}\right)+S 2\left(Z_{3}\right)+S 4\left(Z_{0}\right)\right] \oplus \\
S 0\left[S 2\left(Z_{4}^{\prime}\right)+S 3\left(Z_{5}^{\prime}\right)+S 4\left(Z_{6}^{\prime}\right)+S 1\left(Z_{7}^{\prime}\right)+\right. \\
\left.S 2\left(Z_{1}^{\prime}\right)+S 4\left(Z_{2}^{\prime}\right)+S 2\left(Z_{3}^{\prime}\right)+S 4\left(Z_{0}^{\prime}\right)\right], \\
W_{6} \oplus W_{6}^{\prime}=S 0\left[S 3\left(Z_{4}\right)+S 4\left(Z_{5}\right)+S 1\left(Z_{6}\right)+S 2\left(Z_{7}\right)+\right. \\
\left.S 3\left(Z_{2}\right)+S 1\left(Z_{3}\right)+S 3\left(Z_{0}\right)+S 1\left(Z_{1}\right)\right] \oplus \\
S 0\left[S 3\left(Z_{4}^{\prime}\right)+S 4\left(Z_{5}^{\prime}\right)+S 1\left(Z_{6}^{\prime}\right)+S 2\left(Z_{7}^{\prime}\right)+\right. \\
\left.S 3\left(Z_{2}^{\prime}\right)+S 1\left(Z_{3}^{\prime}\right)+S 3\left(Z_{0}^{\prime}\right)+S 1\left(Z_{1}^{\prime}\right)\right], \\
W_{7} \oplus W_{7}^{\prime}=S 0\left[S 4\left(Z_{4}\right)+S 1\left(Z_{5}\right)+S 2\left(Z_{6}\right)+S 3\left(Z_{7}\right)+\right. \\
\left.S 4\left(Z_{3}\right)+S 2\left(Z_{0}\right)+S 4\left(Z_{1}\right)+S 1\left(Z_{2}\right)\right] \oplus \\
S 0\left[S 4\left(Z_{4}^{\prime}\right)+S 1\left(Z_{5}^{\prime}\right)+S 2\left(Z_{6}^{\prime}\right)+S 3\left(Z_{7}^{\prime}\right)+\right. \\
\left.S 4\left(Z_{3}^{\prime}\right)+S 2\left(Z_{0}^{\prime}\right)+S 4\left(Z_{1}^{\prime}\right)+S 1\left(Z_{2}^{\prime}\right)\right]
\end{array}
$$

What we can hope is to find the eight $Z_{i}$ and eight $Z_{i}^{\prime}$. To this end, we may have to use the above set of equations. Due to the first nonlinear layer and the modulo $p_{0}$ and $p_{1}$ operations in the two cyclic counters, the relation between $Z_{i}$ and $Z_{i}^{\prime}$ should be nonlinear, involving also 16 unknowns $k_{i}$. The above set of 8 equations has 16 unknowns $Z_{i}$ and $Z_{i}^{\prime}$, and they are highly nonlinear. We do not see an easy way to solve this set of equations. The brute-force method needs $2^{128}$ tries. On the other hand, even if one could find the solutions, the number of solutions could be too large. Thus, the difference approach can get rid of the last key-addition layer, but may not go further.

\section{With respect to correlation attacks}

There are different kinds of correlation attacks, such as those in $[1,9]$, but they are only for special stream ciphers. It is impossible to sort out all correlation attacks on a system, but the essential idea of a correlation attack would be to find a relation between output keystream characters and some part or whole of the key, or a relation between some intermediate variables. The purpose of finding such a relation is to get information about the key from known keystream blocks. A way to protect a stream cipher from correlation attacks is to use correlation-immune functions in the system in a proper way.

There are mainly two layers that could protect the system from such an attack. The first is the linear permutation layer described by (1), where each function is correlation-immune of order 6 [10]. The second is the linear compression layer described by (3), where each function is correlation immune of order 7 . These correlation-immune functions and layers are expected to protect the cipher from correlation-immune attacks. 


\section{With respect to affine approximation attacks}

The idea of an affine approximation attack would be to use the best affine approximation (BAA) of some nonlinear components of the system to replace the nonlinear parts, in order to construct a pseudo-keystream generator which produces an output sequence that matches the original keystream sequence with high probability or to recover the key of the original keystream generator. Such an attack, carried out for two kinds of stream ciphers in [2], should not work on our algorithm, due to the high nonlinearity of the S-boxes and the two diffusion layers.

The most reasonable affine approximation of the nonlinear S-boxes is to use affine functions $a x+b$ over $Z_{256}$ to approximate the five S-boxes. However, with a simple $\mathrm{C}$ program we have obtained the following result.

Proposition 3. Let $\operatorname{Pr}$ denote the probability. Then

$$
\begin{aligned}
& \max _{0 \leq a \leq 2550 \leq b \leq 255} \max \operatorname{Pr}(S 0(x)=a x+b)=\frac{2}{256} \\
& \max _{0 \leq a \leq 255} \max _{0 \leq b \leq 255} \operatorname{Pr}(S 1(x)=a x+b)=\frac{3}{256} \\
& \max _{0 \leq a \leq 255} \max _{0 \leq b \leq 255} \operatorname{Pr}(S 2(x)=a x+b)=\frac{3}{256} \\
& \max _{0 \leq a \leq 255} \max _{0 \leq b \leq 255} \operatorname{Pr}(S 3(x)=a x+b)=\frac{3}{256} \\
& \max _{0 \leq a \leq 255} \max _{0 \leq b \leq 255} \operatorname{Pr}(S 4(x)=a x+b)=\frac{4}{256} .
\end{aligned}
$$

This result shows that affine approximations of the S-boxes with $a x+b$ over $Z_{256}$ are very poor. Clearly, every permutation $P(x)$ of $Z_{256}$ can be identified as a permutation $P^{\prime}(y)$ of $Z_{2}^{8}$, and one might therefore be concerned with the affine approximation of $P^{\prime}(y)$ with respect to Boolean affine functions over $Z_{2}^{8}$. However, as our operations in the ciphering algorithm are almost totally based on those of $Z_{256}$, such an affine approximation might not work.

\section{Performance of the algorithm}

On a Pentium ( $75 \mathrm{MHz}$ ) an initial $\mathrm{C}$ code (Borland $\mathrm{C}++$ compiler, version 1991) of the TWOPRIME runs at $1 \mathrm{Mbyte} / \mathrm{sec}$. The test is done with a self-feeding 4 Mbyte input data. An optimized code should run faster.

\section{TWOPRIME-1: A faster version}

It is noted that good linear layers within this ciphering structure is relatively expensive. One way to get a faster version of this algorithm is to use the following simpler linear compression function $C(x)$, but keep other parts the same as 
before:

$$
\left\{\begin{array}{l}
Y_{0}=X_{0}+X_{5}+X_{10}+X_{15} \\
Y_{1}=X_{1}+X_{6}+X_{11}+X_{12} \\
Y_{2}=X_{2}+X_{7}+X_{8}+X_{13} \\
Y_{3}=X_{3}+X_{4}+X_{9}+X_{14} \\
Y_{4}=X_{16}+X_{21}+X_{26}+X_{31} \\
Y_{5}=X_{17}+X_{22}+X_{27}+X_{28} \\
Y_{6}=X_{18}+X_{23}+X_{24}+X_{29} \\
Y_{7}=X_{19}+X_{20}+X_{25}+X_{30}
\end{array}\right.
$$

This linear compression function is less powerful than the original one, and its component functions are correlation-immune of order 3 . We would refer to this faster version as "TWOPRIME-1". A C code of TWOPRIME-1 runs at 1.3 Mbytes/sec on the same machine.

We do not see that TWOPRIME-1 is significantly weaker than TWOPRIME. In fact, we have not found a weakness of TWOPRIME-1, although it might have some.

\section{ONEPRIME: A variant for 64-bit machines}

A variant of TWOPRIME is the ONEPRIME for 64-bit machines. ONEPRIME has only one $(p, a)$ cyclic counter, in which $p$ is the closest 64 -bit prime to $2^{64}-1$ such that $\operatorname{ord}_{p}(2) \geq 2^{32}$, and $a$ is any prime that is approximately $3 p / 4$. Thus, only the first layer of TWOPRIME is modified and others remain the same. The content of the register of the $(p, a)$ cyclic is similarly divided into 8 bytes which are used as the input of the next layer.

A possible choice of the prime $p$ is

$$
p=18446744073709551557=2^{64}-59 \text {. }
$$

Thus,

$$
p-1=2^{2} \times 11 \times 137 \times 547 \times 5594472617641 .
$$

The order of 2 modulo $p$ can be computed with a 64-bit computer, basing on this factorization.

We have about the same theoretical results for ONEPRIME, but have not tested ONEPRIME for performance. However it is clearly much faster than TWOPRIME, as the first layer of ONEPRIME is much faster than that of TWOPRIME.

The security of ONEPRIME and TWOPRIME should be at the same level. The choice between the two algorithms depends on the machines used. For 64machines we would recommend ONEPRIME, as it is faster. We have emphasized on TWOPRIME, as 32-bit machines are mostly used for the time being. 


\section{Other versions}

In some applications, the available memory is very limited. ONEPRIME, TWOPRIME and their variants can be modified by using only one or two nonlinear S-boxes, instead of five. Accordingly, the linear expansion and compression layers should be modified too.

Acknowledgements: The authors would like to thank the three anonymous referees and Adi Shamir for their helpful comments.

\section{References}

1. R. J. Anderson, Searching for optimum correlation attacks, in Fast Software Encryption (B. Preneel, Ed.), LNCS 1008, 137-143.

2. C. Ding, G. Xiao and W. Shan, The Stability Theory of Stream Ciphers, LNCS 561, Springer-Verlag, 1991.

3. C. Ding, Binary cyclotomic generators, in Fast Software Encryption (B. Preneel, Ed.), LNCS 1008, 29-60.

4. J. L. Massey, Shift-register synthesis and $B C H$ decoding, IEEE Trans. Inform. Theory, Vol. IT-15 (1969), 122-127.

5. J. L. Massey, SAFER K-64: A byte-oriented block-ciphering algorithm, in Fast Software Encryption (R. Anderson, Ed.), LNCS 809, Springer-Verlag, 1-17.

6. V. Rijmen, J. Daemen, B. Preneel, and E. De Win, The cipher SHARK, in Fast Software Encryption (D. Gollmann Ed.), LNCS 1039, Springer-Verlag, 99-112.

7. J. A. Reeds, N. J. A. Sloane, Shift-register synthesis (modulo m), SIAM J. Comput. 14, No. 3 (1985), 505-513.

8. P. Rogaway and D. Coppersmith, A Software-Optimized Encryption Algorithm, in Fast Software Encryption (R. Anderson, Ed.), LNCS 809, Springer-Verlag, 1994, 56-63.

9. T. Siegenthaler, Decrypting a class of stream ciphers using ciphertext only, IEEE Trans. Computers, Vol. C-034 (1984), 81-85.

10. T. Siegenthaler, Correlation-immunity of nonlinear combining functions for cryptographic applications, IEEE Trans. Information Theory, Vol. IT-30 (1984), 776-780.

\section{Appendix A: Test values}

$\begin{array}{lrrrrrrrrr}\text { key } & = & 0 & 0 & 0 & 0 & 0 & 0 & 0 & 0 \\ & & 0 & 0 & 0 & 0 & 0 & 0 & 0 & 0 \\ \text { plaintext } & = & 0 & 0 & 0 & 0 & 0 & 0 & 0 & 0 \\ \text { ciphertext } & = & 67 & 67 & 42 & 228 & 183 & 104 & 38 & 130 \\ & & & & & & & & & \\ \text { key } & = & 1 & 1 & 1 & 1 & 1 & 1 & 1 & 1 \\ & & 1 & 1 & 1 & 1 & 1 & 1 & 1 & 1 \\ \text { plaintext } & = & 0 & 0 & 0 & 0 & 0 & 0 & 0 & 0 \\ \text { ciphertext } & = & 21 & 166 & 110 & 21 & 135 & 244 & 34 & 80\end{array}$




\begin{tabular}{|c|c|c|c|c|c|c|c|c|c|}
\hline key & $=$ & 1 & 2 & 3 & 4 & 5 & 6 & 7 & 8 \\
\hline & & 8 & 7 & 6 & 5 & 4 & 3 & 2 & 1 \\
\hline plaintext & $=$ & 0 & 0 & 0 & 0 & 0 & 0 & 0 & 0 \\
\hline ertext & $=$ & 100 & 145 & 30 & 150 & 24 & 92 & 140 & 18 \\
\hline
\end{tabular}

\section{Appendix B: C code of the algorithm}

The following is a $\mathrm{C}$ code of the algorithm for testing the performance. The input is a 4-Mbyte self-feeding data, i.e., only 8-byte inputs are given and the next input block is the output of the previous block.

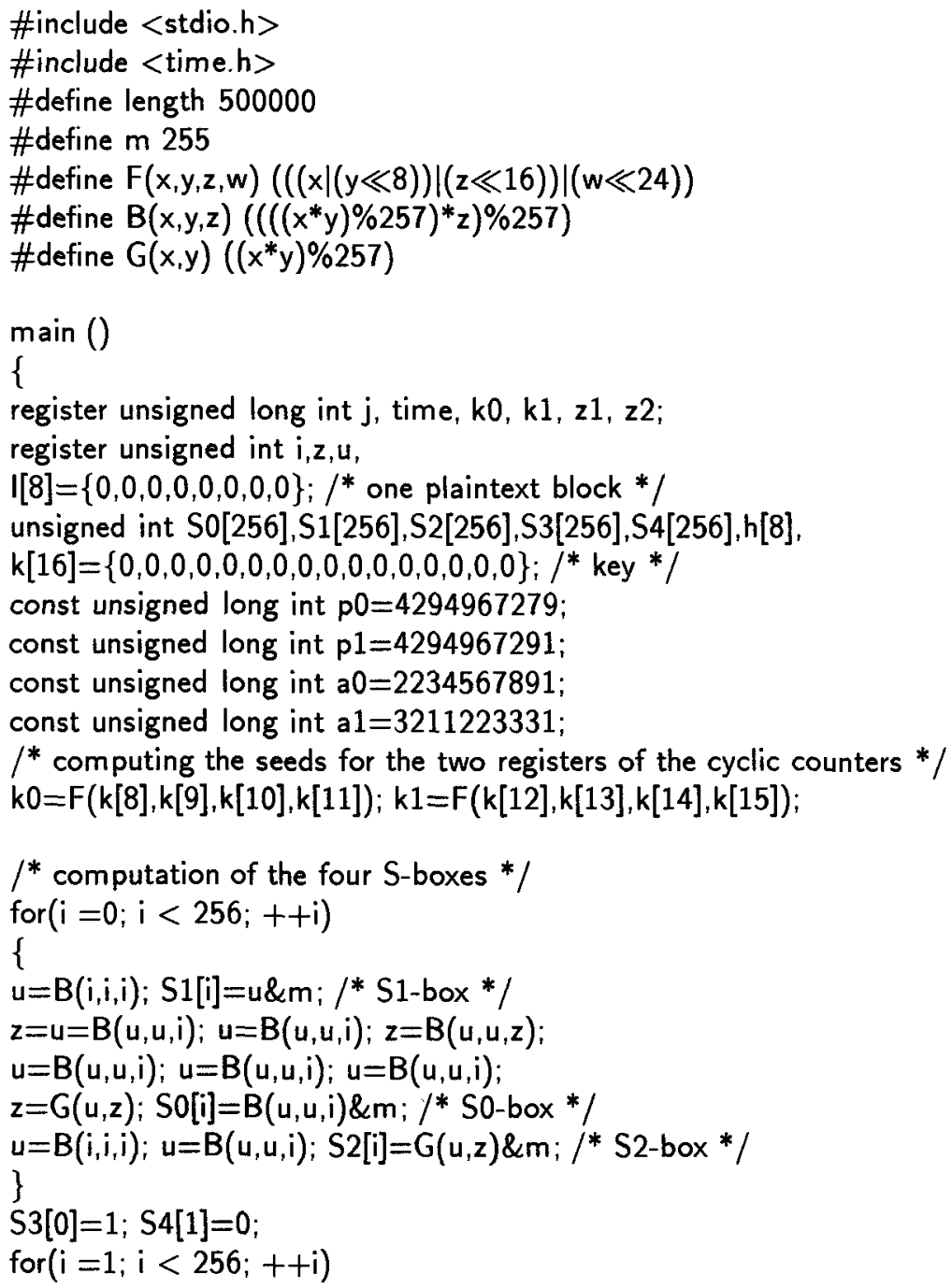




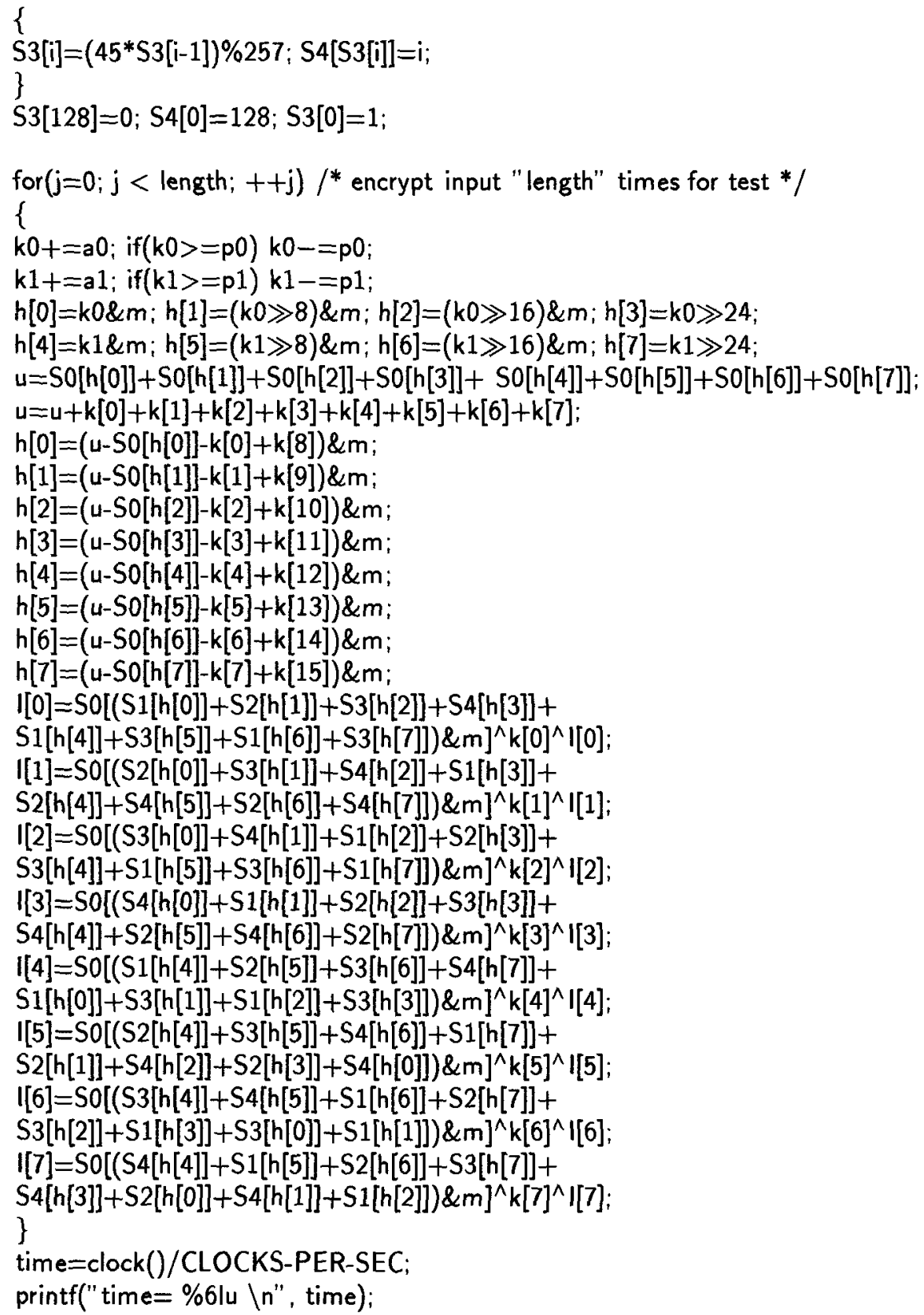

